\title{
Non-Hermitian Broad Aperture Semiconductor Lasers Based on PT-Symmetry
}

\author{
Muriel Botey ${ }^{*}$, , Waqas W. Ahmed ${ }^{2}$, Judit Medina ${ }^{1}$, Ramon Herrero ${ }^{1}$, and Kestutis Staliunas ${ }^{1,3}$ \\ ${ }^{1}$ Departament de Física, Universitat Politècnica de Catalunya, Catalonia \\ ${ }^{2}$ Division of Computer, Electrical and Mathematical Sciences and Engineering, King Abdullah University of \\ Science and Technology (KAUST), Thuwal, 23955-6900, Saudi Arabia \\ ${ }^{3}$ Institució Catalana de Reserca i Estudis Avançats (ICREA), Catalonia \\ *e-mail: muriel.botey@upc.edu
}

\begin{abstract}
In this paper we propose a novel configuration to regularize the complex spatiotemporal dynamics of broad area lasers into bright light beam. It has recently been shown that arbitrary non-Hermitian optical potentials based on local Parity-Time (PT-) symmetry may tailor and control the flow of light, due to the asymmetric mode coupling. We now provide a comprehensive analysis on how this can be applied to stabilize the emission from broad aperture semiconductor lasers. The mechanism relies on a non-Hermitian configuration of the laser potential achieved by simultaneous spatial modulation of the refractive index and gain-loss profiles. This allows concentrating the light into a bright and narrow output beam. We provide a numerical analysis on Vertical Cavity Surface Emitting lasers and Broad Area Semiconductor Lasers. The results indicate a significant intensity enhancement and concentration of the emitted stabilized beam. The proposed mechanism may be technologically achievable, and it is expected to be applicable to regularize the radiation of other broad aperture and microlasers, which typically emit quite random and irregular light patterns. Besides, the reported concentration effect is universal, and could be extended to random and quasi-periodic background potentials.
\end{abstract}

Keywords: non-Hermiticity, semiconductor lasers, local PT-symmetry, VCSEL.

\section{INTRODUCTION}

Broad semiconductor lasers are compact and efficient coherent light sources used for a large variety of applications ranging from data processing to optical communications. Among such lasers are Vertical-Cavity Surface-Emitting Lasers (VCSELs) and Broad Area Semiconductor (BAS) lasers, where the beam emission direction is perpendicular or parallel to the active region, respectively [1]. While the main advantage of semiconductor micro-lasers, including VCSELs and BAS lasers, is their compactness they are generally unstable. Random fluctuations and spatiotemporal instabilities, arise from modulation instability, degrading the spatial beam quality and laser coherence [2]. This instability and the lack of an intrinsic transverse mode control gives rise to different filamentation regimes. In addition, carrier hole burning leads to irregular pulsating patterns [3]. Worth to mention that common techniques to regularize such lasers generally rely on optical feedback which, besides being only applicable to the specific configurations, it may severely reduce the compactness of the design. Therefore, there is still need for an alternative to common techniques to control the complex dynamics of such lasers.

Besides, nowadays, non-Hermitian spatially modulated materials are becoming a novel and flexible platform to manipulate lightwave dynamics. Pump modulations may suppress spatial instabilities in nonlinear optical systems, particularly in BAS devices and VECSEL [4-6]. A particularly remarkable class of such materials are those holding Parity-Time (PT-) symmetry [7] which implies that the complex refractive index satisfies $n(r)=n^{*}(-r)$. For periodic systems, this condition implies that the real part of $n(r)$ (representing refractive index) and the complex part (gain-loss) are depahsed by $90^{\circ}$. When the gain and index modulation amplitudes are balanced (transition point) the PT-symmetric optical potential holds unidirectional light transport, which leads to unconventional beam dynam [8]. PT-symmetric optical potentials uncover novel physical effects such as unidirectional invisibility, power oscillations, coherent perfect absorption, single mode lasing, among others [9].

Recently, a new class of non-Hermitian potentials where the PT-symmetry condition is met locally [10]. Such systems allow to arbitrarily manipulate the field flow in any desired configuration, where local symmetry broken potentials ensure the local directions of the field flows [11]. In this letter, we propose to apply such nonHermitian potentials to control the spatiotemporal dynamics in BAS lasers and VCSELs, while the unidirectional light coupling is expected to collect the energy from the entire active region and concentrate it around the center to form localized beams.

\section{PT-AXISYMMETRIC VCSELS}

The spatiotemporal evolution of the slowly varying intracavity field, $E$ and carrier density, $N$, in VCSELs can be described (neglecting intrinsic absorption) by the system of coupled rate equations [10]: 


$$
\begin{aligned}
& \partial_{t} E(r, t)=-[1+i \theta+2 C(i \alpha-1)(N-1)] E+i \nabla_{\perp}^{2} E+i V(r) E \\
& \partial_{t} N(r, t)=-\gamma\left[N-I_{p}+|E|^{2}(N-1)\right]+\gamma d \nabla_{\perp}^{2} N
\end{aligned}
$$

where all the constants definitions may be found in Ref. [11] and we assume a particular local PT-symmetric potential holding PT-axisymmetry, namely: $V(r)=m_{R} \cos (q r)-i m_{I} \cos (q r-\phi)$ where $q$ is the wavenumber, $m_{\mathrm{I}}$ and $m_{\mathrm{R}}$ are the amplitudes of the index and gain modulations respectively and $\phi$ the relative phase respectively. Indeed, for particular parameters the typical complex and instable VCSEL's emission of Fig.1a is regularized into a narrow beam as shown in Fig. $1 \mathrm{~b}$, where the intensity profile after stationary state indicates that light is strongly concentrated at $r=0$, due to the favoured radial coupling. The corresponding transverse Poynting vector, obtained as $F=i\left(E \nabla E^{*}-E^{*} \nabla E\right)$, see the inset of Fig. $2 \mathrm{~b}$, shows that the wave flux follows a sink pattern. Therefore, the unidirectional inward radial coupling leads to the field concentration around the centre, at $r=0$. For a deeper inspection, we map the concentration factor defined as: $C_{f}=I(x=0) /\langle I(x)\rangle$-being $I$ the intensity-, for a given value of $m_{\mathrm{R}}=0.5$ and different values of $m_{\mathrm{I}}$ and $\phi$. As expected, maximum concentration is found for $\phi \sim 90$ while PT-axisymmetry radially couples light outwards for $\phi=270$, and no concentration is observed. Moreover, this concentration coincides with a stationary state while for other parameters pulsating temporal dynamic regimes are found.

$$
\text { a) }
$$

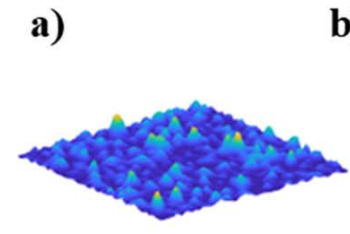

b)
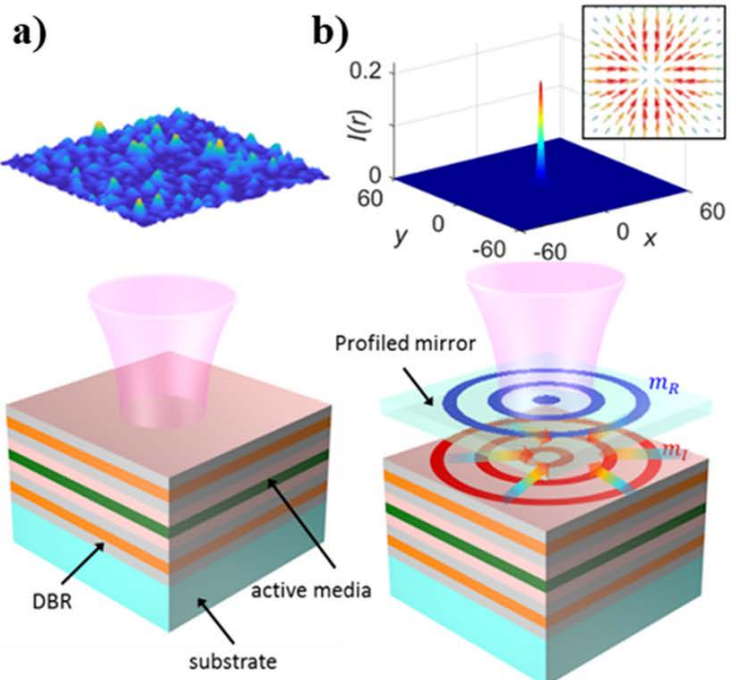

c) $n(r)=m_{R} \cos (q r)-i m_{I} \cos (q r-\phi)$

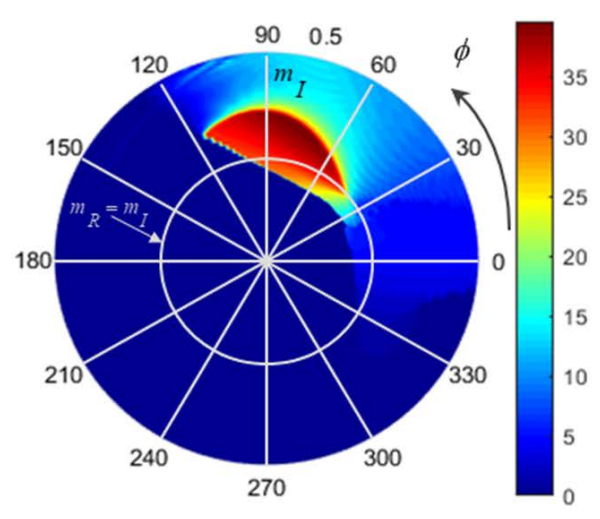

Figure 1: (a) Schematic representation and irregular intensity emission profile of a conventional broad-area VCSELs; (b) Schematics of the proposed PT-axisymmetric VCSELs (blue: index modulation, red: gain modulation) and calculated corresponding narrow and bright emission. The inset depicts the transverse field flow of the stationary regime, leading to energy enhancement and concentration at the center; (c) Axial field concentration in parameter space for a fixed index modulation amplitude, depending on the gain modulation amplitude (radial coordinate) and PT-symmetry phase (angular coordinate) for a 1D simulation, similar results are found for $2 D[13]$.

\section{LOCAL PT-SYMMETRIC BAS}

To analyze the dynamics of local PT-symmetric BAS lasers with we consider both the temporal evolution and the spatial propagation of the electric field, and temporal evolution carriers. We assume that the cavity round trip time is short as compared to the carrier's relaxation time, and that the time evolution of the field in one roundtrip may be calculated by its propagation along the cavity assuming constant carriers. Then, applying the slowly varying envelope approximation for the electric field, the BAS source may be represented by the following nonlinear system of equations for the field amplitude envelope, $A$, and carriers, $N$ :

$$
\begin{aligned}
& \frac{\partial A}{\partial z}=i\left(2 k_{0} n\right) \frac{\partial^{2} A}{\partial x^{2}}+s[(1-i h) N-(1+\alpha)] A+i \Delta n(x, z) k_{0} \\
& \frac{\partial N}{\partial t}=\gamma\left(-N-(N-1)|A|+p_{0}+\Delta p(x, z)+D \nabla^{2} N\right)
\end{aligned}
$$

where $k_{0}$ is the wavevector, $n$ refractive index, $s$ a parameter inversely proportional to the light matter interaction length, $h$ is the Henry factor of the semiconductor, $\alpha$ corresponds to losses, $\gamma$ I's the carriers relaxation rate, $p_{0}$ is the pump, and $D$ is the carrier diffusion. We assume a symmetric harmonic transverse modulation of the pump in the form: $\Delta p(x, z)=m_{1} \cos (q|x|+\Phi)$ where $\mathrm{q}$ is the wavevector, $m_{1}$ the amplitude of the pump modulation and $\Phi$ defines the character of the symmetry axis at $x=0$. Such pump modulation induces an 
in-phase refractive index modulation trough the Henry factor. Therefore, we also introduce a modulation of the refractive index, intended to compensate such index modulation while introducing an overall local PT-symmetric potential with symmetry axis at $x=0$. The index modulations has the form: $\Delta n(x, \mathrm{z})=m_{2} \sin (q|x|+\Phi)+m_{3}$ $\cos (q|x|+\Phi)$. The local PT symmetric potential is intended to concentrate the generated light in the active layer around the symmetry axis while regularizing the emission of the Bas laser. Thus, the unstable emission from a conventional BAS laser, as shown in Fig. 2a, is stabilized into a narrow and bright beam, see Fig. 2b. For particular parameters of the pump and index modulations the radiation is stabilized both spatially, see Fig. 2c, and temporally as shown in Fig. 2d. The insets of both figures provide, for comparison, the same field intensity distribution within the cavity and temporal emission evolution for an unmodulated laser [14].

a)

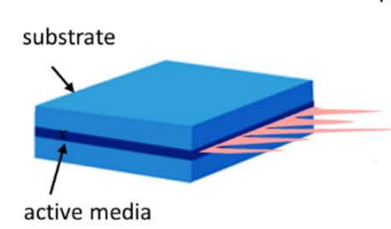

b)

pump modulation

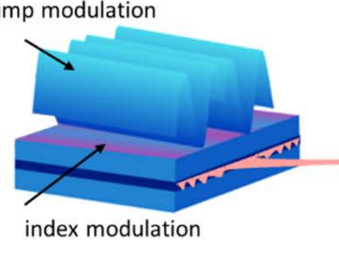

c)

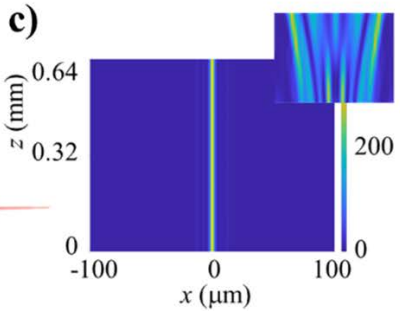

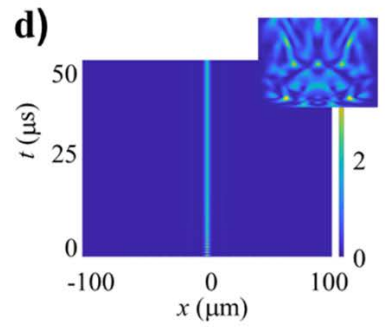

Figure 2: (a) Schematic representation and irregular intensity emission profile from a conventional BAS laser; (b) Schematics of the proposed modulated BAS laser with PT-symmetry with pump and index modulation leading to a bright and narrow emission; (c) Intensity distribution within the BAS laser; (d) Temporal evolution of the output intensity profile of the modulated BAS laser. The insets show the same intensity profiles for an unmodulated BAS laser. $m_{1}=0.5, m_{2}=0.0048$ and $m_{3}=-0.0143$ with $p_{0}=1.23, \Phi=\pi / 4$.

\section{CONCLUSIONS}

To conclude, we propose a feasible and compact scheme tailor and control the complex spatial dynamics of VCSELs and BAS lasers with non-Hermitian potentials. The asymmetric inward coupling concentrates the light around the center rending broad semiconductor lasers into bright and narrow-beam sources. In the case of VCSELS we determine the maximum central intensity and concentration regimes by exploring the modulation parameters and find a significant intensity enhancement and concentration in the emitted beam when the coupling between transverse modes is inwards yet not fulfilling perfect local PT-symmetry. The study of VSELs uncovers rich possibilities for various configurations which could be extended beyond periodic non-Hermitian potentials assuming different random, quasi-periodic complex profiles of the background potential. Also, for the case of BAS the emission may be stabilized both temporally and spatially while the light generated on the active layer is gathered into a narrow central beam by local PT-symmetry. Moreover, the proposed scheme is shown to be robust, compact and efficient and may be applicable to other BAS lasers and microlasers to improve their performance.

\section{ACKNOWLEDGEMENTS}

King Abdullah University of Science and Technology (KAUST) Office of Sponsored Research (OSR-2016CRG5-2950); KAUST Baseline Research Fund (BAS/1/1626-01-01); Spanish Ministerio de Economía y Competitividad (FIS2015-65998-C2-1-P); European Union Horizon 2020 Framework EUROSTARS (E10524 HIP-Laser).

\section{REFERENCES}

[1] G. P. Agrawal and N. K. Dutta, "Semiconductor Lasers," Springer, 2013.

[2] J. Othsubo, "Semiconductor Lasers: Stability, Instability and Chaos," Springer, Heidelberg, 2013.

[3] E. Gehrig and O. Hess, "Spatio-Temporal Dynamics and Quantum Fluctuations in Semiconductor Lasers," Springer, 2013.

[4] R. Herrero, M. Botey, M. Radziunas, and K. Staliunas, "Beam shaping in spatially modulated broad-area semiconductor amplifiers,” Opt. Lett., vol. 37, pp. 5253-5255, 2012.

[5] S. Kumar, R. Herrero, M. Botey, and K. Staliunas, "Suppression of modulation instability in broad area semiconductor amplifiers," Opt. Lett., vol. 39, pp. 5598-5601, 2014.

[6] W. W. Ahmed, S. Kumar, R. Herrero, M. Botey, M. Radziunas, and K. Staliunas, "Stabilization of flatmirror vertical-external-cavity surface emitting lasers by spatiotemporal," ed. G. P. Agrawal, and N. K. Dutta, Semiconductor Lasers, Springer, 2013.

[7] C. M. Bender and S. Boettcher, "Real spectra in non-Hermitian Hamiltonians having PT symmetry," Phys. Rev. Lett., vol. 80, p.5243, 1998. 
[8] C. E. Ruter, K. G. Makris, R. El-Ganainy, D. N. Christodoulides, M. Segev, and D. Kip, "Observation of parity-time symmetry in optics," Nat. Phys., vol. 6, pp. 192-195, 2010.

[9] R. El Gananaiy, K. G. Makris, M. Khajavikhan, Z. H. Musslimani, S. Rotter, and D. N. Chistodoulides, "Non-Hermitian physics and PT-symmetry, "Nature Physics, vol. 14, p. 11, 2018.

[10] W. W. Ahmed, R. Herrero, M. Botey, and K. Staliunas, "Locally parity-time symmetric and globally parity-symmetric systems," Phys. Rev. A, vol. 94, p. 053819, 2016.

[11] W. W. Ahmed, R. Herrero, M. Botey, Z. Hayran, H. Kurt, and K. Staliunas, "Directionality fields generated by a local Hilbert transform," Phys. Rev. A, vol. 97, p. 033824, 2018.

[12] L. Spinelli, G. Tissoni, M. Brambilla, F. Prati, and L. A. Lugiato, "Spatial solitons in semiconductor microcavities," Phys. Rev. A, vol. 58, p. 2542, 1998.

[13] W. W. Ahmed, R. Herrero, M. Botey, Y. Wu, and K. Staliunas, "Regularization of vertical-cavity surfaceemitting laser's emission by periodic non-Hermitian potentials,” arXiv: 1902.04915 [physics.optics], 2019.

[14] J. Medina, R, Herrero, M. Botey, and K. Staliunas, "Spatiotemporal stabilization of locally PT-symmetric BAS lasers", submitted. 